Изв. АН Эૅтонии. Физ. Матем., 1989, 38, № 4, 390-402

удк 532.529

А. КАРТУШИНСКИИ, А. МУЛЬГИ

\title{
МЕЛКОДИСПЕРСНОЕ ТУРБУЛЕНТНОЕ ТЕЧЕНИЕ В ОСЕСИММЕТРИЧНОМ КАНАЛЕ
}

\author{
(Представил Н. Алумяэ)
}

\section{1. Модельное представление}

Исследуется поведение мелкодисперсного потока типа газ-твердые частицы в осесимметричном канале с учетом взаимодействия частиц со стенками канала. Двухфазный поток описывается в рамках представления о взаимопроникающих континуумах $\left[{ }^{1,2}\right]$. Система уравнений для газовой и дисперсной фаз рассматривается в приближении двухфазного турбулентного пограничного слоя и описывается параболическими уравнениями. Хорошо известна общая форма их записи $[3,4]$, за исключением вывода уравнения радиального переноса импульса дисперсной фазы, где в правой части его добавлен член, учитывающий особенности взаимодействия частиц со стенками канала и их диффузию от стенки в поток. Замыкание системы уравнений турбулентного пограничного слоя осуществляется на базе $k$ - $\varepsilon$-модели, которая учитывает влияние стенки с помощью дополнительных членов в пристеночной области. Связь пульсационных параметров газовой и дисперсной фаз осуществляется с использованием формул, полученных в рамках модели [5]. Взаимодействие фаз в осредненном движении определяется силами сопротивления и Магнуса. Появление последней обусловлено скоростным скольжением фаз (разности скоростей газовой и дисперсной фаз) и вращением частиц дисперсной фазы под действием градиента скорости несущей среды. Қак показывают численные расчеты, из-за больших градиентов скорости газа вблизи стенок возникают существенные радиальные потоки на стенку. Демпфирующее действие на эти потоки примеси вблизи стенок оказывает учитываемый диффузионный член, появляющийся в уравнении переноса импульса дисперсной фазы в радиальном направлении. Благодаря ему с увеличением концентрации частиц на стенке создается противоположное их рассеивание - диффузия частиц в поток. Учесть вращение, приобретаемое за счет соударений частиц со стенками канала в рамках представления о взаимопроникающих континуумах, по-видимому, невозможно. Подобное вращение можно учесть лишь рассматривая движение индивидуальных частиц, мигрирующих от стенки к стенке. Очевидно, что в потоке находятся частицы с различной ориентацией вращения. Проводя осреднение различно ориентированных частиц по их ансамблю, можно, следовательно, предположить, что в среднем вращательный импульс столкновительного происхождения самонивелируется.

Движение мелкодисперсной примеси на установившемся участке анализируется в $\left[{ }^{6,7}\right]$. Пульсационное скольжение фаз и влияние примеси на турбулентность несущей среды рассчитываются в этих работах по-разному и полученные результаты отличаются от данных расчетов. В $\left[{ }^{6}\right]$ используется $k$ - $\varepsilon$-модель с учетом поправочных членов, 
характеризующих дисперсность среды в уравнениях переноса $k$ й $\varepsilon$ соответственно. В $\left[{ }^{7}\right]$ пульсационная скорость - скорость миграции частицы - находится в результате решения уравнения пульсационного смещения частицы в поле среды, которое моделируется моногармоническими пульсациями с частотой, равной частоте ее энергоемких пульсаций. В предложенной системе уравнений двухфазного пограничного слоя в канале [ $\left.{ }^{7}\right]$ не ясно каким образом учитывается влияние примеси на турбулентность среды через турбулентные характеристики - видимо, как-то через турбулентную вязкость. Влияние примеси учитывается только через силу сопротивления, которая деформирует поля осевой осредненной скорости газовой среды. Спорным является вычисление радиальной скорости дисперсной фазы как суммирование отдельных составляющих ее, вызванных различными механизмами взаимодействия с несущей средой и стенками канала, причем, неясным является моделирование дисперсной среды в радиальном направлении в целом движением одиночной частицы в этом направлении. Е. П. Медников $\left[{ }^{7}\right]$ касается механизма сальтации частиц со стенками и недостаточно подробно его характеризует, в частности, коэффициент восстановления скорости частицы при отскоке. Непонятно, почему отсутствует в поле зрения статьи вращательный импульс частицы при ее отскоке от стенки.

В отличие от $\left[{ }^{6,7}\right]$ в данной работе проводится исследование динамики распределения параметров газовой и дисперсной фаз на всем участке движения частиц, включая разгонный, в начале которого частицы примеси, вводятся с практически нулевой начальной скоростью. Расчеты охватывают и стабилизированный участок течения, где в зависимости от физических параметров (размера частиц, их плотности материала, чисел Рейнольдса) наблюдается движение примеси со стабильным постоянным отставанием от газовой фазы [8].

Из-за малости гравитационная сила в расчетах не учитывалась.

\section{2. Начальные условия для газовой и дисперсной фаз}

Для решения задачи о двухфазном турбулентном потоке в канале необходимо предварительно иметь информацию об однофазном турбулентном потоке. Мелкодисперсная примесь попадает в уже сформировавшийся однофазный поток, где затем происходит развитие и установление двухфазного течения. При этом полученные значения скоростей $u, v$, перепада давления $d p / d x$, характеристик турбулентности $k, \varepsilon, v_{t}, L$ однофазного турбулентного потока на установившемся участке канала принимаются за начальные поля для газовой части двухфазного турбулентного течения. Для нахождения параметров потока на установившемся участке решалась система уравнений однофазного турбулентного пограничного слоя, которая представляет собой систему параболических уравнений и двух квадратур. Совместное решение уравнений неразрывности и переноса импульса газа в осевом направлении, как показано в $\left[{ }^{9}\right]$, дает выражение для поперечной скорости газа в квадратурах (уравнение (6)), а для нахождения градиента давления (уравнение (5)) можно воспользоваться, как и в [9 $]$, условием непроницаемости на стенке, где $v=0$. Система уравнений в безразмерном виде тогда будет следующей:

$u \frac{\partial u}{\partial x}+v \frac{\partial u}{\partial r}=\frac{1}{r} \frac{\partial}{\partial r} r\left(v_{t}+v\right) \frac{\partial u}{\partial r}-\frac{\partial p}{\partial x}$,

$u \frac{\partial k}{\partial x}+v \frac{\partial k}{\partial r}=\frac{1}{r} \frac{\partial}{\partial r} r\left(\frac{v_{t}}{\sigma_{k}}+v\right) \frac{\partial k}{\partial r}+v_{t}\left(\frac{\partial u}{\partial r}{ }^{2}-\varepsilon-2 v\left(\frac{\partial \sqrt{k}}{\partial r}\right)^{2}\right.$, 
$u \frac{\partial \varepsilon}{\partial x}+v \frac{\partial \varepsilon}{\partial r}=\frac{1}{r} \frac{\partial}{\partial r} r\left(\frac{v_{t}}{\sigma_{\varepsilon}}+v\right) \frac{\partial \varepsilon}{\partial r}+c_{\varepsilon 1} \frac{\varepsilon}{k} v_{t}\left(\frac{\partial u}{\partial r}\right)^{2}-c_{e 2 t} \frac{\varepsilon^{2}}{k}+$ $+2 v v_{t}\left(\frac{\partial^{2} u}{\partial r^{2}}\right)^{2}$

$v_{t}=c_{\mu} k^{2} / \varepsilon$

$\frac{d p}{d x}=\int_{0}^{1} \frac{1}{u^{2}} \frac{\partial}{\partial r}\left(r\left(v_{t}+v\right) \frac{\partial u}{\partial r}\right) d r\left[\int_{0}^{1} r u^{-2} d r\right]^{-1}$,

$v=\frac{u}{r}\left[-\int_{0}^{r} \frac{1}{u^{2}} \frac{\partial}{\partial r}\left(r\left(v_{t}+v\right) \frac{\partial u}{\partial r}\right) d r+\frac{d p}{d x} \int_{0}^{r} \frac{r d r}{u^{2}}\right]$.

Обезразмеривающими параметрами являются радиус канала $R$ и динамическая скорость $v_{*}$. Коэффициенты $\sigma_{k}=1, \quad \sigma_{\varepsilon}=1,3, \quad c_{\varepsilon 1}=$ $=1,43, \quad c_{\mu}=0,09 \quad-$ числовые постоянные, а $c_{\mu t}=$ $=c_{\mu} \cdot \exp \left(-2,5 /\left(1+\left(\frac{R_{T}}{50}\right)\right)\right), c_{22 t}=2\left(1-0,3 \exp \left(-R_{T}^{2}\right)\right)-$ пристеночные функции, зависящие от турбулентного числа Рейнольдса $R_{T}=\frac{k^{2}}{v \varepsilon}\left[{ }^{10}\right]$. Система уравнений $(1)-(6)$ решалась при следующих граничных условиях

условие симметрии задачи

$$
r=0, \frac{\partial u}{\partial r}=\frac{\partial k}{\partial r}=\frac{\partial \varepsilon}{\partial r}=v=0,
$$

условия прилипания и непроницае- $r=1, u=k=\varepsilon=v=0$. мости стенки

При решении системы уравнений (1)-(6) с граничными условиями (7) - (8) используется трехслойная схема турбулентного пограничного слоя: турбулентное ядро, переходный слой и вязкий подслой. Аналитическое задание начальных условий для осевой скорости газа по трехслойной схеме известно [11]. Для аналитического задания турбулентной вязкости в начальном сечении воспользуемся формулой Прандтля, в которой длину пути перемешивания определим по формуле Кутателадзе $\left[{ }^{12}\right]$. При выводе аналитических соотношений для турбулентной энергии $k$ и скорости ее диссипации $\varepsilon$ воспользуемся известным соотношением между турбулентным трением и турбулентной энергией для свободных течений $\left[{ }^{10}\right]$ и применим его для пристенного слоя с учетом пристеночных функций. Таким образом, можно задать аналитически все искомые величины: $u, k, \varepsilon, v_{t}$ в начальном сечении при $x=0$. Система уравнений (1)-(3) записывалась в конечных разностях с использованием шеститочечного шаблона по монотонной схеме Самарского $\left[{ }^{13}\right]$. Нелинейность преодолевалась взятием итераций с предыдущего шага. Использование трехслойной схемы требует переменного шага по радиальной координате. В ходе численных расчетов проводился контроль по балансовому соотношению сохранения расхода газа: $\int_{0}^{1} r u d r=$ const. Относительная вычислительная погрешность не превышала $2 \%$ и связана с применением неконсервативной численной схемы. Как показывают расчеты, масштаб турбулентности $L=\frac{k \sqrt{k}}{\varepsilon}$ намного превосходит используемую расчетную сетку. Выход на стабилизированный режим наблюдался на расстояниях порядка 50 калибров. Таким 
образом, полученные скорости в осевом и радиальном направлениях $(u, v)$, перепад давления $\left(\frac{d p}{d x}\right), \quad$ характеристики турбулентности $\left(k, \varepsilon, v_{t}, L\right)$ на стабилизированном участке использовались как начальные параметры для газовой фазы при исследовании двухфазного турбулентного течения в канале.

Начальные распределения осевой скорости дисперсной фазы задаются в предположении, что мелкодисперсная примесь полностью следует пульсациям газа и начинает двигаться с пульсационной скоростью газа, определяемой интенсивностью турбулентности, т. е. $u_{s}(r)=\sqrt{\frac{2}{3} k(r)}$. Для определения начальной радиальной скорости дисперсной фазы используется балансовое соотношение в радиальном направлении

$$
B \equiv \frac{c_{D}\left(v-v_{s}\right) \sqrt{\left(u-u_{s}\right)^{2}+\left(v-v_{s}\right)^{2}}}{\bar{\delta}}+\frac{\partial u}{\partial r}\left(u-u_{s}\right)=0
$$

т. е. предполагается, что сила Магнуса, под действием которой частицы начинают совершать движение поперек канала, уравновешивается силой сопротивления, действующей на частицы со стороны несущей среды в этом направлении, откуда

$$
v_{s}(r)=v(r)+\frac{\frac{\partial u}{\partial r}\left(u-u_{s}\right) \bar{\delta}}{c_{D} \sqrt{\left(u-u_{s}\right)^{2}+\left(v-v_{s}\right)^{2}}}
$$

Әто уравнение решается итеративным методом. Начальное распределение относительной массовой концентрации находится из балансового соотношения между миграционным и диффузионным потоками массы примеси в радиальном направлении, т. е. делается предположение о справедливости соотношения $\alpha v_{s}=\left(D_{s}+v\right) \frac{\partial \alpha}{\partial r}$, которое на самом деле должно быть справедливым лишь на стабилизированном участке течения $\left[{ }^{8}\right]$. Решая это уравнение, получим распределение концентрации в начальном сечении типа $\alpha(r)=\alpha_{0} \exp \left(\frac{v_{s} r}{D_{s}+v}\right)$. Для нахождения неизвестной величины $\alpha_{0}$ воспользуемся законом сохранения массы примеси $\int_{0}^{1} r \alpha u_{s} d r=$ const. Поскольку расходная концентрация $x-$ величина наперед заданная, то, определяя ее как $\quad x=\int_{0}^{1} r \alpha u_{s} d r / \int_{0}^{1} r u d r$, можно найти постоянную $\alpha_{0}$, характеризующую абсолютную величину массовой концентрации, например, в центральной точке профиля концентрации, т. е. $\alpha_{0}=x \cdot u_{0} \int_{0}^{1} r \tilde{u} d r / u_{s 0} \int_{0}^{1} r \tilde{\alpha} \tilde{u}_{s} d r$. Подставляя полученное выражение для $\alpha_{0}$ в выражение для концентрации $\alpha(r)$, получим поле концентрации в начальном сечении

$$
\alpha(r)=x \frac{u_{0} \int_{0}^{1} r \tilde{u} d r}{u_{s 0} \int_{0}^{1} r \tilde{\alpha} \tilde{u}_{s} d r} \exp \left(\frac{v_{s} r}{D_{s}+v}\right) .
$$


При этом, как показывают расчеты, величина массовой концентрации в начальном сечении больше на порядок своей величины на установившемся участке течения при заданной величине начальной осевой скорости частиц. Это вытекает из закона сохранения массы примеси. Таким образом, начальные поля скоростей дисперсной фазы и концентрации будут иметь вид

$$
\begin{gathered}
u_{s}(r)=\sqrt{\frac{2}{3} k(r)}, \\
v_{s}(r)=v(r)+\frac{\frac{\partial u}{\partial r}\left(u-u_{s}\right) \bar{\delta}}{c_{D} \sqrt{\left(u-u_{s}\right)^{2}+\left(v-v_{s}\right)^{2}}}, \\
\alpha(r)=x \frac{u_{0} \int_{0}^{1} r \tilde{u} d r}{u_{s 0} \int_{0}^{1} r \tilde{\alpha} \tilde{u}_{s} d r} \cdot \exp \left(\frac{v_{s} r}{D_{s}+v}\right),
\end{gathered}
$$

где коэффициент сопротивления частицы $c_{D}$ определяется согласно $\left[{ }^{14}\right]$ как $\quad c_{D}=\frac{22,8}{\operatorname{Re}_{p}}+\frac{6,63}{\sqrt{\operatorname{Re}_{p}}}+0,333$, а число Рейнольдса - по частице $\operatorname{Re}_{p}$ как $\operatorname{Re}_{p}=\sqrt{\left(u-u_{s}\right)^{2}+\left(v-v_{s}\right)^{2}} 2 \bar{\delta} \operatorname{Re}_{*}$. Здесь, как и для параметров чистого газа, все физические величины обезразмерены. Величина $\bar{\delta}$ - относительный диаметр частицы $\bar{\delta}=\frac{\delta}{2 R}, \quad \mathrm{Re}_{*}-$ число Рейнольдса, определяемое по динамической скорости $v_{*}$ как $\operatorname{Re}_{*}=\frac{v_{*} R}{v}$. Индекс 0 относится к центральной точке потока, а тильда над параметрами $u, \alpha, u_{s}$ указывает на относительность данной величины, отнесенной к своей величине в центральной точке профиля.

\section{3. Система уравнений и граничные условия двухфазного турбулентного трубного пограничного слоя}

Система уравнений двухфазного турбулентного течения в канале записывается в приближении турбулентного пограничного слоя и представляет собой систему уравнений параболического типа. Она описывает процессы, происходящие на разгонном и стабилизированном участках. Если совместно решить уравнение неразрывности и переноса импульса газовой фазы вдоль осевой координаты, а также учесть условие непроницаемости стенки, то можно, как в $\left[{ }^{9}\right]$, получить выражение для перепада давления и радиальной скорости газовой фазы - уравнения (22), (23). Уравнение (23) представляет собой интегральное уравнение Вольтерра второго рода и для его решения воспользуемся схемой из $\left[{ }^{15}\right]$. В безразмерном виде (обезразмеривающие параметры указаны в разделе 2) система уравнений двухфазного турбулентного трубного пограничного слоя выглядит

$$
\begin{array}{r}
A=\frac{c_{D}\left(u-u_{s}\right) \sqrt{\left(u-u_{s}\right)^{2}+\left(v-v_{s}\right)^{2}}}{\bar{\delta}}-\frac{\partial u}{\partial r}\left(v-v_{s}\right), \\
u \frac{\partial u}{\partial x}+v \frac{\partial u}{\partial r}=\frac{1}{r} \frac{\partial}{\partial r} r\left(v_{t}^{\prime}+v\right) \frac{\partial u}{\partial r}-\frac{\partial p}{\partial x}-\frac{3}{8} \frac{\varrho}{\varrho p} \alpha A,
\end{array}
$$




$$
\begin{aligned}
& u \frac{\partial k}{\partial x}+v \frac{\partial k}{\partial r}=\frac{1}{r} \frac{\partial}{\partial r} r\left(\frac{v_{t}}{\sigma_{k}}+v\right) \frac{\partial k}{\partial r}+v_{t}\left(\frac{\partial u}{\partial r}\right)^{2}-\varepsilon-2 v\left(\frac{\partial \sqrt{k}}{\partial r}\right)^{2}, \\
& u \frac{\partial \varepsilon}{\partial x}+v \frac{\partial \varepsilon}{\partial r}=\frac{1}{r} \frac{\partial}{\partial r} r\left(\frac{v_{t}}{\sigma_{\varepsilon}}+v\right) \frac{\partial \varepsilon}{\partial r}+c_{\varepsilon 1} \frac{\varepsilon}{k} v_{t}\left(\frac{\partial u}{\partial r}\right)^{2}- \\
& -c_{\varepsilon 2 t} \frac{\varepsilon^{2}}{k}+2 v v_{t}\left(\frac{\partial^{2} u}{\partial r^{2}}\right)^{2} \\
& u_{s} \frac{\partial u_{s}}{\partial x}+\left(v_{s}-\frac{\left(D_{s}+v_{s t}+2 v\right)}{\alpha} \frac{\partial \alpha}{\partial r}\right) \frac{\partial u_{s}}{\partial r}=\frac{1}{r} \frac{\partial}{\partial r} r\left(v_{s t}+v\right) \frac{\partial u_{s}}{\partial r}+\frac{3}{8} \frac{\varrho}{\varrho p} A \text {, } \\
& u_{s} \frac{\partial v_{s}}{\partial x}+\left(v_{s}-\frac{\left(2 D_{s}+v_{s t}+3 v\right)}{\alpha}\right) \frac{\partial v_{s}}{\partial r}=1 \frac{1}{r} \frac{\partial}{\partial r} r\left(v_{s t}+v\right) \frac{\partial v_{s}}{\partial r}+\frac{3}{8} \frac{\varrho}{\varrho p} B+ \\
& +\frac{v_{s}}{\alpha} \frac{1}{r} \frac{\partial}{\partial r} r\left(D_{s}+v\right) \frac{\partial \alpha}{\partial r} \\
& u_{s} \frac{\partial \alpha}{\partial x}+v_{s} \frac{\partial \alpha}{\partial r}=\frac{1}{r} \frac{\partial}{\partial r} r\left(D_{s}+v\right) \frac{\partial \alpha}{\partial r}-\alpha\left(\frac{\partial u_{s}}{\partial x}+1 \frac{1}{r} \frac{\partial}{\partial r} r v_{s}\right) \\
& v_{t}=c_{\mu t} k^{2} / \varepsilon, \\
& \frac{d p}{d x}=\int_{0}^{1} \frac{1}{u^{2}} \frac{\partial}{\partial r}\left(r\left(v_{t}^{\prime}+v\right) d r\left[\int_{0}^{1} r u^{-2} d r\right]^{-1}-\right. \\
& -\frac{3}{8} \frac{\varrho}{\varrho p} \int_{0}^{1} \frac{\alpha r d r}{u^{2}} A\left[\int_{0}^{1} r u^{-2} d r\right]^{-1} \\
& v=\frac{u}{r}\left[-\int_{0}^{r} \frac{1}{u^{2}} \frac{\partial}{\partial r}\left(r\left(v_{t}^{\prime}+v\right) \frac{\partial u}{\partial r}\right) d r+\frac{d p}{d x} \int_{0}^{r} \frac{r d r}{u^{2}}+\right. \\
& \left.+\frac{3}{8} \frac{\varrho}{\varrho p} \int_{a}^{r} \frac{\alpha r d r}{u^{2}} A\right] \text {. }
\end{aligned}
$$

Уравнения (18)-(20) записаны в предположении пренебрежения продольными корреляциями $\bar{\alpha} \overline{u_{s}^{\prime} u_{s}^{\prime}}, \overline{\alpha^{\prime} u_{s}^{\prime}}$ по сравнению с поперечными . $\bar{\alpha} \overline{u_{s}^{\prime} v_{s}^{\prime}}, \overline{\alpha^{\prime} v_{s}^{\prime}}$, пренебрежением корреляций по осевой координате, а также отбрасыванием тройных корреляций $\overline{\alpha^{\prime} u_{s}^{\prime} u_{s}^{\prime}}, \overline{\alpha^{\prime} v_{s}^{\prime} v_{s}^{\prime}}$ и т. д. Особый интерес представляет последний член в правой части уравнения (19). Он характеризует диффузионный перенос импульса в радиальномнаправлении и наряду с осредненным переносом импульса в данном направлении под действием сил сопротивления и Магнуса играет существенную роль вблизи стенок канала, где примесь имеет тенденцию скапливаться. Этот диффузионный «силовой» член своим воздействием как бы препятствует «бесконечному» накапливанию частиц на стенках. Входящий в систему уравнений коэффициент турбулентной вязкости газовой фазы $v_{t}^{\prime}$ корректируется по формуле $\left[{ }^{5}\right]$, учитывающей влияние примеси на турбулентность, и для полностью увлекаемых пульсациями 
газа мелких частиц определяется как $v_{t}^{\prime}=\frac{v_{t}}{(1+\alpha)^{2}}$. Величины $D_{s}$ и $v_{s t}$ - соответственно коэффициенты диффузии примеси и псевдотурбулентной вязкости дисперсной фазы, представляемой сплошной средой. По формулам [5] для мелких, полностью увлекаемых пульсациями газа частиц эти коэффициенты можно представить как $D_{s}=\frac{v_{t}}{(1+\alpha)^{2}}$, $v_{s t}=\frac{v_{t}}{(1+\alpha)^{2}}$. Остальные коэффициенты $c_{\mu t}, c_{D}$ и т. д. определены в разделе 2. Система параболических уравнений (15)-(20) решалась при следующих граничных условиях для газовой фазы:

условие симметрии

$$
\begin{aligned}
& r=0, \frac{\partial u}{\partial r}=\frac{\partial k}{\partial r}=\frac{\partial \varepsilon}{\partial r}=v=0, \\
& r=1, u=k=\varepsilon=v=0,
\end{aligned}
$$

условия прилипания и
непроницаемости стенки

Для дисперсной фазы:

условие симметрии

$$
r=0, \frac{\partial u_{s}}{\partial r}=\frac{\partial \alpha}{\partial r}=v_{s}=0
$$

условие скольжения дисперной фазы вдоль стенки

условие непроницаемости стенки условие отсутствия потока массы примеси на стенку, вытекающее из условия непроницаемости стенки

$$
r=1,\left\{\begin{array}{l}
\frac{\partial u_{s}}{\partial r}=0 \\
v_{s}=0 \\
\frac{\partial \alpha}{\partial r}=0 .
\end{array}\right.
$$

Физически условие скольжения дисперсной фазы вдоль стенки означает, что частицы ведут себя не совсем как сплошная среда, они напоминают скорее разреженный газ, где при больших числах Кнудсена для молекул не наблюдается эффект прилипания к поверхности тел при их обтекании, а наблюдается эффект их проскальзывания. Кроме того, из-за достаточной разреженности частиц в самой дисперсной фазе отсутствует давление, характерное для несущей газовой фазы. В канале продольный перепад давления определяется градиентом скорости газа на стенке. Если по аналогии с газовой фазой задать условие прилипания дисперсной фазы на стенке, то вдоль канала должен присутствовать градиент давления дисперсной фазы, но его нет. Использование условия скольжения было связано с соблюдением законов сохранения массы газовой фазы $\int_{0}^{1} r u d r=$ const и массы примеси $\int_{0}^{1} r a u_{s} d r=\mathrm{const}$, относительная погрешность в вычислении которых составляла менее $5 \%$. Вычислительные погрешности связаны с неконсервативностью численной схемы и наличием схемной вязкости, проявляющейся отчасти в ламинарном подслое, где турбулентная вязкость мала. Численная схема решения параболических уравнений аналогична описанной в разделе 2. Следует обратить внимание на то, что при решении уравнений двухфазного турбулентного трубного пограничного слоя под знаком вторых производных по радиальной координате при описании дисперсной фазы по аналогии с газовой наряду с турбулентной вязкостью или ее аналогами присутствует ламинарная вязкость. Если отбросить величину ламинарной вязкости под знаком вторых производных в дисперсной части системы уравнений, то в ламинарном подслое, где все турбулентные коэффициенты или их аналоги становятся достаточно малыми, уравнения пограничного слоя (18)-(23) - уравнения параболического 
типа - теряют свой смысл, исчезает вторая производная и уравнения преобразуются в уравнения типа газовой динамики.

\section{4. Результаты}

Расчетный материал проиллюстрирован на рис. $1-8$, где представлены распределения скоростей газовой и дисперсной фаз, концентрации примеси и турбулетных характеристик газовой фазы (энергии, скорости ее диссипации, вязкости и макромасштаба) в турбулентном ядре, переходном слое и ламинарном подслое для конкретного примера расчета двухфазного турбулентного трубного пограничного слоя с мелкими частицами размером $\delta=16$ мкм с расходной концентрацией примеси $x=$ $=1$ кг/кг при средней скорости потока $u=50 \mathrm{~m} /$ с в канале диаметром $D=16$ мм. Для наглядности переходный слой и ламинарный подслой выделены в отдельные рисунки (четная нумерация) из-за существенной градиентности полей в этих областях. Результаты расчетов двухфазного турбулентного пограничного слоя на установившемся участке сравниваются с результатами расчетов в начальном сечении с целью прослеживания динамики развития двухфазного течения в осесимметричном канале. Согласно численным расчетам мелкодисперсного трубного течения, выход на стабилизированный установившийся режим происходит вниз по потоку на расстояниях более 100 калибров. Начальные поля газовой фазы, являющиеся одновременно полями распределения параметров чистого трубного потока (пунктирные кривые на рисунках), удовлетворительно описываются экспериментальными данными $[11,16,17]$. На рис. 1,2 изображены профили осевых скоростей газовой и дисперсной фаз $u, u_{s}$, обезразмеренные величиной осевой

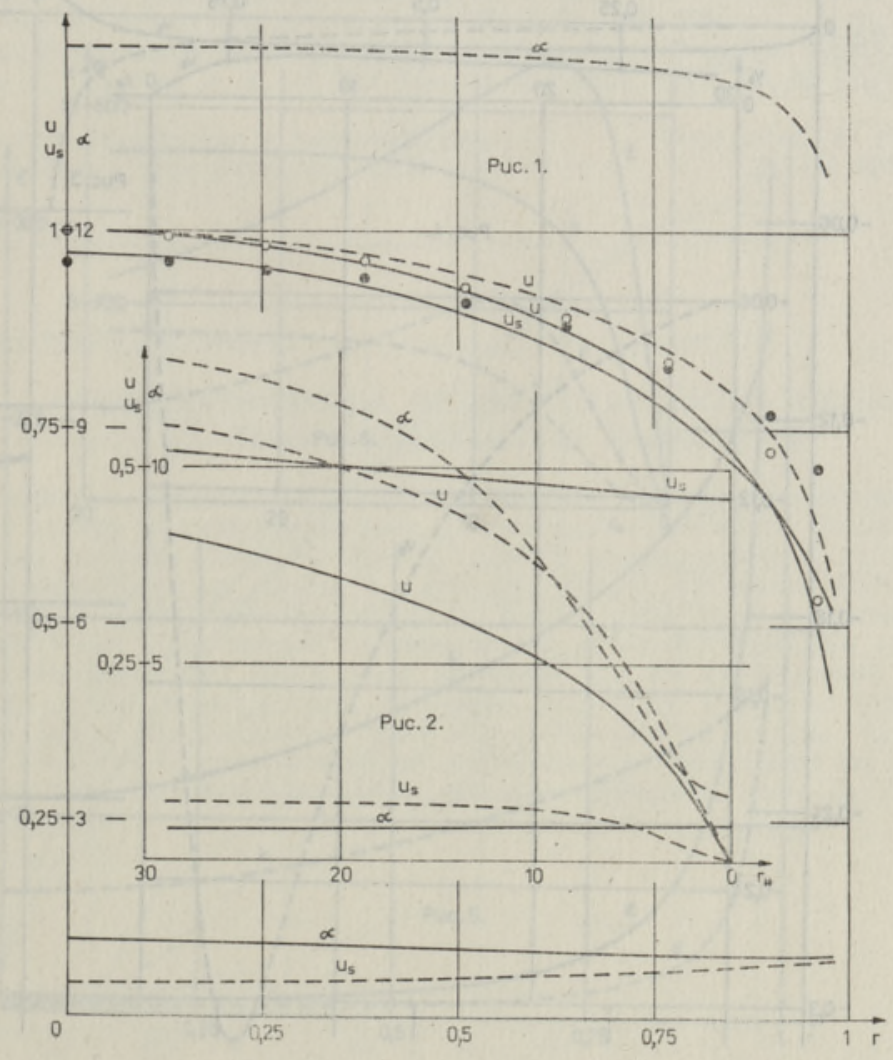


скорости газовой фазы в начальном сечении потока в центральной ее точке, и концентрации примеси $\alpha$, а также проведено сопоставление с экспериментом в турбулентной части, в турбулентном ядре потока (см. рис. 1, где экспериментальные точки $\bigcirc$ относятся к осевой скорости газовой фазы, точки - к осевой скорости дисперсной фазы). Незначительная деформация профиля осевой скорости газовой фазы (кривые $u$, рис. 1,2$)$ при существенном изменении относительной массовой концентрации примеси (более чем на порядок (кривые $\alpha$ на тех же рисунках)) указывает на то, что закон сохранения массы газа в канале выполняется, а это означает правильность выбранного модельного представления. По модельному представлению частицы попадают в поток со скоростью, определяемой интенсивностью турбулентности несущей среды, т. е. почти с нулевой скоростью (пунктирная кривая $u_{s}$ на рис. 1), а это согласно закону сохранения массы примеси приводит к существенному увеличению относительной массовой концентрации частиц в начальном сечении канала (пунктирная кривая $\alpha$ на рис. 1 ). Ускоряясь, в основном за счет силы сопротивления, мелкие частицы почти догоняют газ и в турбулентном ядре форма профиля их осевой скорости (сплошная кривая $u_{s}$ на рис. 1) приобретает форму профиля, близкую к форме профиля осевой скорости газовой фазы (сплошная кривая $u$ на рис. 1). Наблюдается лишь незначительное скольжение фаз (разность скоростей) в осевом направлении. В переходном слое и особенно в вязком подслое наблюдается заметная неравновесность течения газовой и дисперсной фаз (сплошные кривые $u$ и $u_{s}$ на рис. 2) благодаря

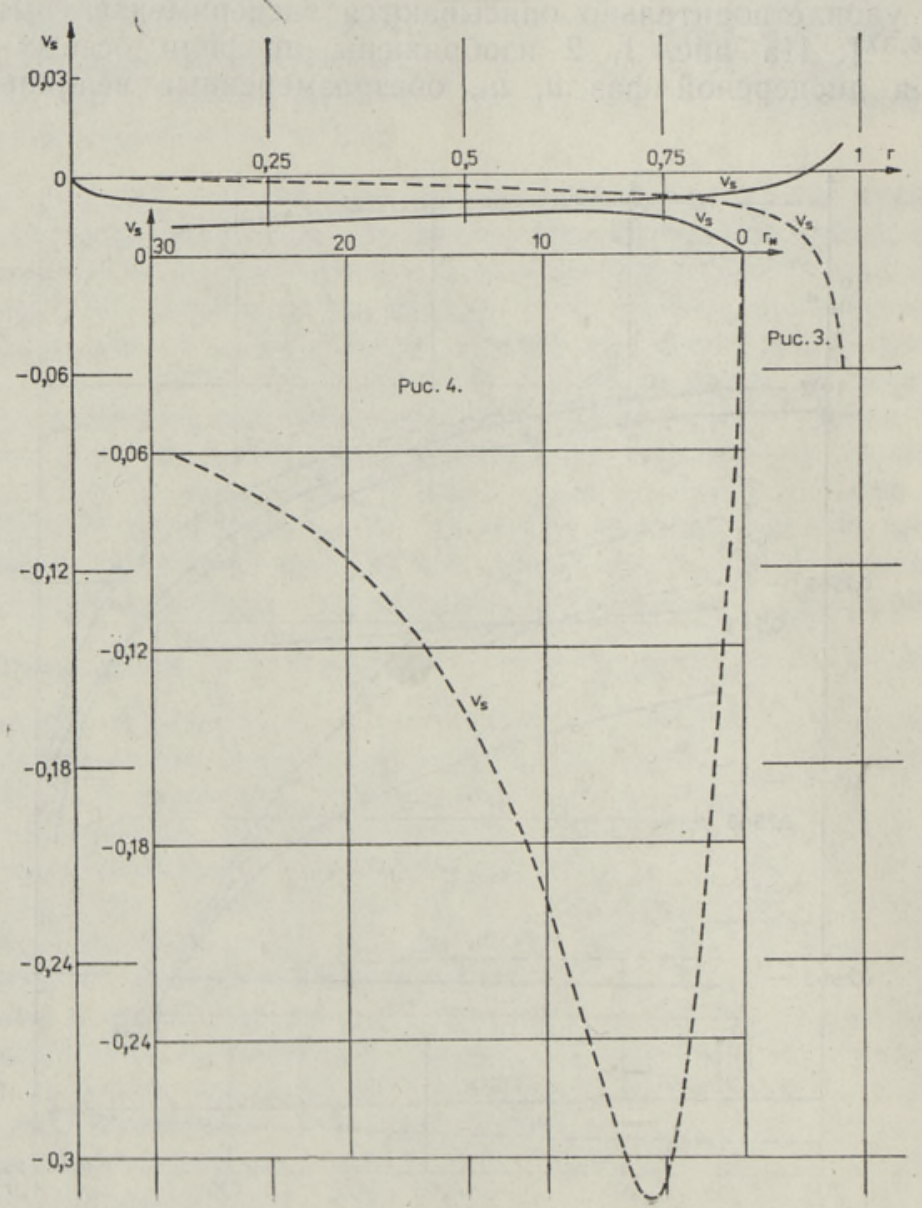


инерционности частиц и поэтому в этих областях для фаз ставятся различные граничные условия: для газа - условие Дирихле, т. е. условие прилипания на стенке, для дисперсной фазы - условие Неймана, т. е. условие скольжения вдоль стенки. По мере разгона частиц в канале их относительная массовая концентрация уменьшается и на стаби лизированном участке профиль концентрации приближается к равномерному как в турбулентном ядре (сплошная кривая $\alpha$ на рис. 1 ), так и в переходном слое и вязком подслое (сплошная кривая $\alpha$ на рис. 2). Этого следовало ожидать, так как и для скорости дисперсной фазы и для концентрации примеси были заданы однотипные граничные условия градиентного типа Неймана (в задаче равные нулю). Подтверждением правильности модельного представления является также выполнение условия сохранения расхода массы примеси. Относительная погрешность в вычислении интегральных характеристик расхода газа и расхода массы примеси на протяжении исследуемого участка длиною 172 калибра составляла менее 5\%. Распределение знакопеременной величины радиальной скорости дисперсной фазы показано на рис. 3 в турбулентном ядре и на рис. 4 - в переходном слое и ламинарном подслое. Отсутствие сопоставления данной величины с аналогичной радиальной скоростью газовой фазы в указанных областях связано с малостью последней, величина которой более чем на 2 порядка меньше скорости частиц. Как видно из рис. 3,4 , наиболее интересные события развиваются в пристеночной области (рис. 4), где существуют большие градиенты скорости несущей среды, и следовательно, на частицы действует большая сила Магнуса. Начальное распределение радиальной скорости частиц получено из балансового соотношения между силами сопротивления и Магнуса, спроецированными на радиальное направление. В процессе вычислений радиальной скорости частиц учитывается также диффузионный поток частиц от стен-

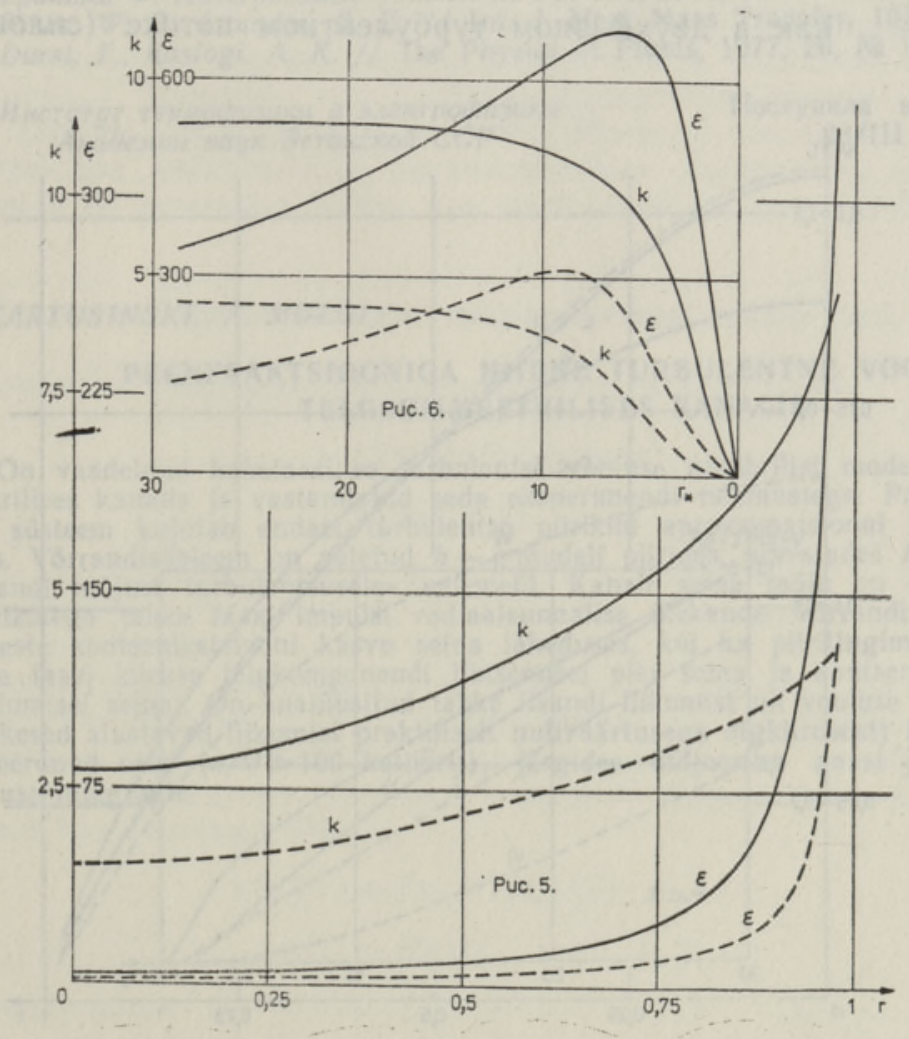


ки, где происходит их накопление из-за положительного направления действия силы Магнуса в пристеночной области, к центру канала. Знакоположительность величины $v_{s}$ в переходном слое и вязком подслое (сплошная кривая $v_{s}$ на рис. 4), таким образом, предопределена направлением действия силы Магнуса в этих областях, величина радиальной скорости частиц в турбулентном ядре имеет противоположное направление (отрицательна), поскольку вызвана отставанием частиц от скорости несущей фазы в этой области (сплошная кривая $v_{s}$ на рис. 3). Сравнивая турбулентные характеристики газовой фазы в двухфазном турбулентном трубном пограничном слое на установившемся участке с их начальными полями, являющимися одновременно полями чистого турбулентного трубного пограничного слоя, видим, что уровень турбулентности (сплошная кривая $k$, рис. 5,6$)$, скорость диссипации турбулентной энергии (сплошная кривая $\varepsilon$, рис. 5,6 ) и турбулентная вязкость (сплошная кривая $v_{t}$, рис. 7,8 ) в двухфазном потоке заметно возрастают и в турбулентном ядре и в пристеночной области. Это на первый взгляд странно, если учесть, что в уравнениях переноса турбулетной энергии $k$ (уравнение (16)) и скорости ее диссипации $\varepsilon$ (уравнение (17)) нет непосредственного влияния примеси на эти турбулентные характеристики. Влияние твердой примеси проявляется через поля скоростей газовой фазы в осевом $u$ и радиальном $v$ направлениях. Начальные поля турбулентных характеристик - энергии (пунктирные кривые $k$, рис. 5,6 ), скорости ее диссипации (пунктирные кривые $\varepsilon$, рис. 5,6 ) и вязкости (пунктирные кривые $v_{t}$, рис. 5,6 ), описывающие однофазный турбулентный трубный пограничный слой согласуются с экспериментальными данными $\left[{ }^{11,16,17}\right]$ как в турбулентном ядре, так и в переходном слое и ламинарном подслое и являются хорошей основой для проведения последующих расчетов в двухфазном турбулентном пограничном слое в канале. Макромасштаб турбулентности $L=\frac{k \sqrt{k}}{\varepsilon}$ как в двухфазном турбулентном потоке (сплошная кри-

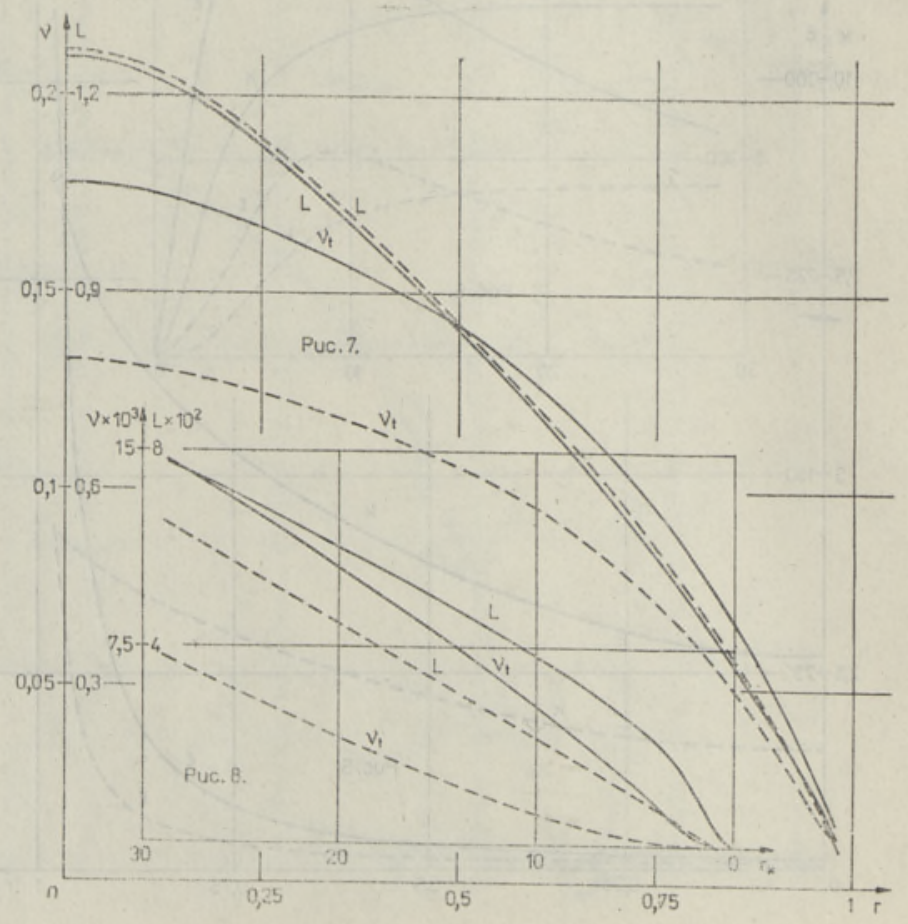


вая $L$, рис. 7,8$)$, так и в «чистом» однофазном турбулентном потоке (пунктирная кривая $L$, рис. 7,8 ), слабо деформируется по всей толщине пограничного слоя. Если обратиться к выражению макромасштаба $L$, и заметить, что в двухфазном турбулентном пограничном слое в канале наблюдается пропорциональный рост как турбулентной энергии $k$, так и скорости ее диссипации $\varepsilon$ по сравнению с однофазным турбулентным трубным пограничным слоем, то величина их отношения $L$, по-видимому, и должна слабо меняться, т. е. оставаться величиной постоянной.

\section{ЛИТ Е РА Т У Р А}

1. Крайко А. Н., Стернин Л. Е. Прикл. матем. и мех., 1965, 29, вып. 3, 418-429.

2. Нигматулин Р. И. // Основы механики гетерогенных сред. М., Наука, 1978.

3. Васильков А. П. // Изв. АН СССР. Мех. жидкости и газа, 1976, № 5, 57-63.

4. Картуиинский А. И. // Изв. АН СССР. Мех. жидкости и газа, 1984, № 1, 36-41.

5. Абрамович Г. Н., Гиршович Т. А. // Парожидкостные потоки. Мннск, 1977, $155-175$.

6. Кондратьев Л. В. // Турбулентные двухфазные течения и техника эксперимента. Таллинн, $1985,144-148$.

7. Медников Е. П. // Метеорология и гидрология, 1985, № 9, 82-94.

8. Мульги А. C. // Турбулентные двухфазные течения и техника эксперимента. Таллннн, $1985,161-167$.

9. Абрамович Г. Н. и др. Теория турбулентных струй. М., Наука, 1984, 716.

10. Моулден Т., Фрост У. Турбулентность. Принципы и применения. М., Мир, 1980.

11. Лойцянский Л. Г. Механика жидкости и газа. М., Наука, 1973, 847.

12. Шлихтине $\Gamma$. Теория пограничного слоя. М., Наука, $1974,711$.

13. Самарский A. А. Введение в теорию разностных схем. М., Наука, 1971, 552.

14. Кравцов Н. В. Инж.-физ. ж., 1968, 15, № 3, 464-470.

15. Трикоми Ф. Интегральные уравнения. М., ИЛ, 1960, 299.

16. Jones, W. P., Launder, B. E. // Int. J. Heat Mass Transfer, 1973, 16, 1119-1130.

17. Durst, F., Rastogi, A. K. // The Physics of Fluids, 1977, 20, № 12, 1975-1985.
Институт термофизики и электрофизики Академии наук Эстонской ССР
Поступила в редакцию
28/III 1988

\section{A. KARTUSINSKI, A. MULGI}

\section{PEENFRAKTSIOONIGA HIIBNE TURBULENTNE VOOLUS TELGSOMMEETRILISES KANALIS}

On vaadeldud kahefaasilise turbulentse vooluse numbrilist modelleerimist telgsümmeetrilises kanalis ja vastandatud seda eksperimendi tulemustega. Paraboolsete vôrrandite süsteem kujutab endast turbulentse piirikihi aproksimatsiooni kolmekihilise skeemina. Vōrrandisüsteem on suletud $k-\varepsilon$-mudeli piirides, arvestades Abramovitši mudeli «Lisandi mõjust turbulentsusele» valemeid. Kanali seina mõju on arvestatud niihästi lisaliikmega tahke faasi impulsi radiaalsuunalise ülekande võrrandis, mis summutab osakeste kontsentratsiooni kasvu seina läheduses, kui ka piiritingimusi arvesse vōttes tahke faasi kiiruse telgkomponendi libisemisel piki seina ja kontsentratsioonigradiendi puudumisel seinal. On analüüsitud tahke lisandi liikumist nii vooluse formeeruvas osas (osakesed alustavad liikumist praktiliselt nullväärtusega algkiirusest) kui ka vooluse stabiliseerunud osas ( $x / D>100$ kaliib̆rit), järgides üldjoontes gaasi ja lisandi masside jäävuse integraale. 


\section{A. KARTUSHINSKI and A. MULGI}

THE MOTION OF FINE SOLID SUSPENSION IN THE TURBULENT GASEOUS FLOW IN AN AXISYMMETRIC CHANNEL

In the present paper the numerical simulation of a two-phase flow in an axisymmetric channel compared to the experimental data is considered. The model is based on a system of parabolic equations with the turbulent boundary-layer approximation (3-layer system). Taking into consideration some formulae from the Abramovich's model that describes the influence of solid particles on the turbulency of the gaseous phase, the system is closed by the $k-\varepsilon$ model. The influence of the channel wall on the radial motion of particles in the dispersed phase that results in damping the increase of particle concentration at the wall, is given by an additional number in the momentum equation. On the other hand, the influence is described by the boundary conditions of the dispersed phase: the slip of the axial velocity component along the channel wall and the absence of the concentration gradient on the wall.

The launching motion of the discrete phase in the instability length of the channel (the initial speed of the particles is practically zero) as well as in the stability length of the channel $(x / D>100)$ is analyzed following the integrals of the particle mass concentration and that of the mass of the gaseous phase. 